\title{
Oscillating spin-2 dark matter
}

\author{
Luca Marzola, Martti Raidal, and Federico R. Urban \\ Laboratory of High Energy and Computational Physics, \\ NICPB, Rävala puiestee 10, 10143 Tallinn, Estonia
}

(Received 2 September 2017; published 8 January 2018)

\begin{abstract}
The negative outcomes of laboratory searches, juxtaposed with cosmological observations, may indicate that dark matter has a gravitational origin. We show that coherent oscillations of a massive spin-2 field emerging from bimetric theory can easily account for the observed dark matter abundance. The framework, based on the only known consistent extension of general relativity to interacting spin-2 fields, is testable in precision measurements of the electric charge variation by means of atomic clocks, molecular systems, dedicated resonant mass detectors, as well as gravity interferometers and axionlike-particle experiments. These searches, therefore, provide a new window into the phenomenology of gravity which complements the results of dedicated tests of gravitation. We also present a multimetric extension of the scenario that straightforwardly implements the clockwork mechanism for gravity, explaining the apparent weakness of this force.
\end{abstract}

DOI: $10.1103 /$ PhysRevD.97.024010

The presence of dark matter (DM) in our Universe is inferred purely from its gravitational effects [1,2], availing the idea that an extended theory of gravity manifests itself in this mysterious component. Although gravity is the only force of nature which presently lacks a quantum description, consistent extensions of general relativity (GR) exist and commonly introduce new gravitational degrees of freedom [3]. It is not yet known whether these new fields could play the role of DM, as their production mechanisms and, most importantly, their phenomenology have not been investigated in detail. In this work, we show that the additional massive spin-2 field predicted by the bimetric theory (BT) of gravity [4,5] provides a natural DM candidate in the form of a coherently oscillating classical configuration, set in motion by the misalignment mechanism [6]. We demonstrate that the scenario complies with the requirement of isotropic cosmological solutions [7] and is consistent with all current observations [8] over a DM mass range $10^{-23}-0.1 \mathrm{eV}$. The framework we propose is directly testable in a variety of atomic, molecular, and nuclear systems sensitive to oscillations of the elementary electric charge [9], including atomic clocks [10,11] and atomic spectroscopy experiments $[12,13]$, dedicated resonant mass detectors $[14,15]$, as well as gravitational wave interferometers [16] and axionlike-particle (ALP) experiments [17-19]. In regard to this, the oscillating spin-2 scenario is distinguishable from ALPs and other DM

Published by the American Physical Society under the terms of the Creative Commons Attribution 4.0 International license. Further distribution of this work must maintain attribution to the author(s) and the published article's title, journal citation, and DOI. Funded by SCOAP ${ }^{3}$. candidates due to its polarization effects, induced by couplings to electric and magnetic fields of the form $E_{i} E_{j} \pm B_{i} B_{j}$ and $E_{i} B_{j} \pm B_{i} E_{j}$, where $i, j \in\{x, y, z\}$. We also find that multimetric extensions of the considered framework [20] predict a stepwise growth of the matter component of Universe during its late evolution, which could be detected by the EUCLID satellite mission [21]. Additionally, this scenario could naturally explain the weakness of gravity via the clockwork mechanism $[22,23]$. We thus propose a viable DM candidate of gravitational origin which possesses nontrivial cosmological implications and allows for direct tests of gravity in a variety of laboratory experiments. If confirmed, these results would strongly deepen our understanding of DM and pave the way for a fuller comprehension of gravity.

In addition to the usual metric field $g_{\mu \nu}$, the BT action includes a second interacting spin-2 field $f_{\mu \nu}$ [4],

$$
\begin{aligned}
S= & -\frac{M_{\mathrm{P}}^{2}}{1+\alpha^{2}} \int \mathrm{d}^{4} x\left[\sqrt{|g|} R(g)+\alpha^{2} \sqrt{|f|} R(f)\right. \\
& \left.+2 \frac{\alpha^{2} M_{\mathrm{P}}^{2}}{1+\alpha^{2}} \sqrt{|g|} V\left(g, f ; \beta_{n}\right)\right]+\int \mathrm{d}^{4} x \sqrt{|g|} \mathcal{L}_{\mathrm{m}}(g, \Psi),
\end{aligned}
$$

where $M_{\mathrm{P}} \approx 2.4 \times 10^{18} \mathrm{GeV}$ is the reduced Planck mass and $\alpha$ is a dimensionless constant that accounts for differences in the strength of the interactions associated to the spin-2 metric fields. Throughout this work, we adopt the (+- - ) signature for both the metrics and allow greek subscripts or superscripts to take values in $\{0,1,2,3\}$; latin indices span instead the restricted set $\{1,2,3\}$. On top of the usual Einstein-Hilbert kinetic 
terms for $f_{\mu \nu}$ and $g_{\mu \nu}$, the first line of Eq. (1) harbors the interaction potential $V\left(g, f ; \beta_{n}\right)$. This contains five dimensionless parameters $\beta_{n}, n \in[0,4]$, and is engineered around the requirement that no propagating ghost degrees of freedom appear in the theory $[4,24,25]$. The last term in Eq. (1) is the Lagrangian of generic matter fields $\Psi$. Notice that the absence of ghost modes forces the latter to interact only with one of the metric fields present in the action $[26,27]$, effectively spoiling its symmetry under the interchange of the two metrics.

In order to disentangle the roles of the two metrics, we linearize the theory by considering metric fluctuations around identical backgrounds $\bar{f}_{\mu \nu}=\bar{g}_{\mu \nu}$,

$$
\begin{aligned}
& g_{\mu \nu}=\bar{g}_{\mu \nu}+\epsilon h_{\mu \nu}, \\
& f_{\mu \nu}=\bar{g}_{\mu \nu}+\epsilon \ell_{\mu \nu},
\end{aligned}
$$

with $\epsilon$ a small expansion parameter $[28,29]$. The resulting quadratic action can be diagonalized by means of the following substitutions:

$$
\begin{gathered}
h_{\mu \nu}=: \frac{1}{M_{\mathrm{P}}}\left(G_{\mu \nu}-\alpha M_{\mu \nu}\right), \\
\ell_{\mu \nu}=: \frac{1}{M_{\mathrm{P}}}\left(G_{\mu \nu}+\alpha^{-1} M_{\mu \nu}\right) .
\end{gathered}
$$

As we can see, the parameter $\alpha$ also quantifies the mixing between the interaction eigenstates $h_{\mu \nu}$ and $\ell_{\mu \nu}$ and the mass eigenstates $G_{\mu \nu}$ and $M_{\mu \nu}$. In terms of $G_{\mu \nu}$ and $M_{\mu \nu}$, we find

$$
\begin{aligned}
S^{(2)}:= & \int \mathrm{d}^{4} x \sqrt{|\bar{g}|}\left[\mathcal{L}_{\mathrm{GR}}^{(2)}(G)+\mathcal{L}_{\mathrm{FP}}^{(2)}(M)\right. \\
& \left.-\frac{1}{M_{\mathrm{P}}}\left(G_{\mu \nu}-\alpha M_{\mu \nu}\right) T^{\mu \nu}(\Psi)\right]+\mathcal{O}\left(\epsilon^{3}\right) .
\end{aligned}
$$

Here, $\mathcal{L}_{\mathrm{GR}}^{(2)}(X)$ is the usual expression obtained by expanding the action of GR at the quadratic level $\mathcal{L}_{\mathrm{GR}}^{(2)}(X):=$ $M_{\mathrm{P}}^{2} X^{\mu \nu} \mathcal{E}_{\mu \nu}^{\lambda \kappa} X_{\lambda \kappa}$, with the Lichnerowicz operator defined as

$$
\begin{aligned}
\mathcal{E}_{\mu \nu}^{\lambda \kappa}:= & \delta_{\kappa}^{\lambda} \square-\mathcal{G}_{\mu \nu} \mathcal{G}^{\lambda \kappa} \square+\mathcal{G}^{\lambda \kappa} \nabla_{\mu} \nabla_{\nu} M \\
& +\mathcal{G}_{\mu \nu} \nabla^{\lambda} \nabla^{\kappa}-2 \nabla^{\lambda} \nabla_{(\mu} \delta_{\nu)}^{\kappa},
\end{aligned}
$$

where $2 X_{(\mu, \nu)}:=X_{\mu \nu}+X_{\nu \mu} . T^{\mu \nu}(\Psi)$ is the stress-energy tensor of the matter fields $\Psi$. Indices are raised and lowered by means of the background metric $\bar{g}_{\mu \nu}$.

The remaining term in Eq. (6), $\mathcal{L}_{\mathrm{FP}}^{(2)}(M)$, is the quadratic Fierz-Pauli Lagrangian

$$
\mathcal{L}_{\mathrm{FP}}^{(2)}(M):=\mathcal{L}_{\mathrm{GR}}^{(2)}(M)-\frac{m_{\mathrm{FP}}^{2}}{4}\left(M_{\mu \nu} M^{\mu \nu}-M^{2}\right),
$$

where we identified the Fierz-Pauli mass $m_{\mathrm{FP}}:=$ $\sqrt{\beta_{1}+2 \beta_{2}+\beta_{3}} M_{\mathrm{P}}$ for the spin-2 field $M_{\mu \nu}$. The two remaining parameters $\beta_{0}$ and $\beta_{4}$ correspond to two identical cosmological constants that, for the purpose of this work, can be safely neglected.

Recasting Eq. (1) in terms of the mass eigenstates $G_{\mu \nu}$ and $M_{\mu \nu}$ makes the field content of the theory explicit. At the linearized level, BT contains a massless spin-2 field, $G_{\mu \nu}$, which possesses two helicity states as does the usual graviton of GR. We also have an additional spin-2 field, $M_{\mu \nu}$, characterized by a Fierz-Pauli mass $m_{\mathrm{FP}}$ induced by the interaction potential $V$. Being massive, $M_{\mu \nu}$ propagates five independent degrees of freedom.

We define a nonlinear effective background $\mathcal{G}_{\mu \nu}$ as

$$
\mathcal{G}_{\mu \nu}=\bar{g}_{\mu \nu}+\frac{1}{M_{\mathrm{P}}} G_{\mu \nu} .
$$

Since terms linear in $M_{\mu \nu}$ and of any order in $G_{\mu \nu}$ vanish in the expansion of the original action (1) [28,29], we can partially resum the expansion to separate the background $\mathcal{G}_{\mu \nu}$ from the dynamics of the massive fluctuation $M_{\mu \nu}$. Through this procedure, we formally obtain

$$
\begin{aligned}
S_{\text {spin-2 }}= & -M_{\mathrm{P}}^{2} \int \mathrm{d}^{4} x \sqrt{|\mathcal{G}|} R(\mathcal{G}) \\
& +\int \mathrm{d}^{4} x \sqrt{|\mathcal{G}|} \mathcal{L}_{\mathrm{FP}}^{(2)}(M)+\mathcal{O}\left(M_{\mu \nu}^{3}\right) .
\end{aligned}
$$

The theory at hand then contains a propagating spin-2 particle, the massive field $M_{\mu \nu}$, on a generic background encoded in the metric $\mathcal{G}_{\mu \nu}$.

The separation of the action presented in Eq. (10) emerges naturally when $H \ll m_{\mathrm{FP}}$, where $H:=\partial \log a(t) / \partial t$ is the Hubble parameter and $a(t)$ is the scale factor of the Universe. The background metric $\mathcal{G}_{\mu \nu}$ has, in fact, a characteristic length and time scale of $1 / H$ which is much longer than the typical wavelength $1 / m_{\mathrm{FP}}$ of the massive spin 2. We limit the values of $\alpha$ and $m_{\mathrm{FP}}$ in a way in which the theory reproduces GR within the sensitivity of gravity tests [30,31]: for small $m_{\mathrm{FP}} \ll 1 \mathrm{meV}$, we take $\alpha \ll 1$, whereas we can allow $\alpha \geq 1$ when $m_{\mathrm{FP}} \gg 1 \mathrm{meV}$.

The equation of motion (EOM) for the massive spin-2 field $M_{\mu \nu}$ can be derived from Eq. (10) via a variational derivative with respect to the field $M^{\mu \nu}$,

$\mathcal{E}_{\mu \nu}^{\lambda \kappa} M_{\lambda \kappa}-R M_{\mu \nu}+\mathcal{G}_{\mu \nu} R^{\lambda \kappa} M_{\lambda \kappa}+\frac{1}{2} m_{\mathrm{FP}}^{2}\left(M_{\mu \nu}-\mathcal{G}_{\mu \nu} M\right)=0$,

where $M:=M_{\mu}^{\mu}$ and indices have been raised and lowered by means of the nonlinear metric $\mathcal{G}_{\mu \nu}$, which also determines the expressions of the metric connection used in the covariant derivative $\nabla_{\mu}$ and curvature tensor. The structure of the EOM (11) can be simplified by taking into account the linearized Bianchi identities, which imply that $\nabla^{\mu} M_{\mu \nu}=\nabla_{\nu} M$, and thus $\nabla^{\mu} \nabla^{\nu} M_{\mu \nu}=\square M$. Substituting this constraint in the trace of (11) results in the tracelessness of the massive spin-2 field, $M=0$, which in turn enforces transversality: $\nabla^{\mu} M_{\mu \nu}=0$. These constraints ensure that 
the massive spin-2 field propagates only 5 degrees of freedom.

BT was extensively studied in limits where the mass of the new spin-2 field is assumed to be of order of the Hubble parameter today [32-37], $m_{\mathrm{FP}} \sim H_{0} \sim 10^{-33} \mathrm{eV}$, to model the late evolution of the Universe. Yet, if regimes relevant for particle physics are instead considered, the framework delivers a suitable DM candidate in the form of spin-2 particles at a mass scale ranging form $\mathrm{MeV}$ to $\mathrm{TeV}$ depending on the production mechanism [28,29,37-39]. Here, we consider a complementary regime of the theory, showing that the observed DM relic density can be explained in terms of the classical dynamics of the spin2 field, by its coherent oscillations, for a wide mass range that is reminiscent of axion or ALP scenarios.

To this purpose, we investigate the dynamics of a sub-eV spin-2 field in the early Universe, considering a Friedmann-Lamaître-Robertson-Walker background $\mathcal{G}_{\mu \nu}=$ $\operatorname{diag}\left(1,-a^{2}(t),-a^{2}(t),-a^{2}(t)\right)$ where $t$ is the cosmic time. Because the stress-energy tensor sourcing the former is necessarily that of a perfect fluid, Eq. (11) reduces to

$$
\square M_{\mu \nu}+2 R_{\mu \alpha \nu \beta} M^{\alpha \beta}+m_{\mathrm{FP}}^{2} M_{\mu \nu}=0,
$$

where we reordered the covariant derivative in the Lichnerowicz operator by introducing the curvature tensor and have accounted for the transversality and tracelessness of $M_{\mu \nu}$. In terms of the EOM for the individual components of the massive spin-2 field, these constraints force $M_{0 \nu}=0$ and $M_{i}^{i}=0$.

The dynamics of the five remaining degrees of freedom, in Fourier space, is then governed by

$$
\ddot{M}_{i j}+3 H \dot{M}_{i j}+k^{2} M_{i j}+m_{\mathrm{FP}}^{2} M_{i j}=0,
$$

where a dot indicates differentiation with respect to $t$. Notice that the above equations do not involve any further approximation.

With the EOM at hand, we focus on the homogeneous modes of the spin-2 field, $k \ll m_{\mathrm{FP}}$, obeying

$$
\ddot{M}_{i j}+3 H \dot{M}_{i j}+m_{\mathrm{FP}}^{2} M_{i j}=0 .
$$

At early times, when $H \gg m_{\mathrm{FP}}$, these modes are essentially massless and frozen by the Hubble friction. This regime is maintained until $H \sim m_{\mathrm{FP}}$, at which point the field begins to oscillate with a characteristic frequency $\omega=m_{\mathrm{FP}}$,

$$
M_{i j} \sim a(t)^{-3 / 2} \cos \left(m_{\mathrm{FP}} t\right) .
$$

The resulting evolution of each component is thus identical to that of a free scalar field oscillating in the quadratic potential imposed by a mass term.

Rapid oscillations around the minimum $\left(m_{\mathrm{FP}} \gg H\right)$ ensure that $M_{i j}$ behaves as matter, as required of a suitable
DM candidate $[19,37]$. This can be shown by computing the energy density $\rho_{\mathrm{DM}}$ and pressure $P_{\mathrm{DM}}$ from the energymomentum tensor of $M_{\mu \nu}$, defined as

$$
T_{M}^{\mu \nu} \equiv \frac{1}{\sqrt{|\mathcal{G}|}} \frac{\delta\left(\sqrt{|\mathcal{G}|} \mathcal{L}_{\mathrm{FP}}^{(2)}(M)\right)}{\delta \mathcal{G}_{\mu \nu}} .
$$

We find $\rho_{\mathrm{DM}} \sim \dot{M}_{i j} \dot{M}^{i j}+m_{\mathrm{FP}}^{2} M_{i j} M^{i j}$ and $P_{\mathrm{DM}} \sim \dot{M}_{i j} \dot{M}^{i j}-$ $m_{\mathrm{FP}}^{2} M_{i j} M^{i j}$. A direct computation shows that in the fast oscillating regime $\dot{M}_{i j} \dot{M}^{i j}=m_{\mathrm{FP}}^{2} M_{i j} M^{i j}$ and the pressure term therefore vanishes. Hence, from the Bianchi identities,

$$
\nabla_{\mu} T_{M}^{\mu}{ }_{\nu}=0
$$

and by assuming a perfect fluid form for $T_{M^{\mu}{ }_{\nu}=}=$ $\operatorname{diag}\left(\rho_{\mathrm{M}},-P_{\mathrm{M}},-P_{\mathrm{M}},-P_{\mathrm{M}}\right)$, we immediately infer that $\dot{\rho}_{\mathrm{M}}+3 H \rho_{\mathrm{M}}=0$, in an analogy with the ALPs case.

Since the oscillating massive spin-2 field behaves like matter, we estimate its contribution to the observed DM density to be [19]

$$
\Omega_{\mathrm{DM}} \approx 2.0\left(\frac{m_{\mathrm{FP}}}{10^{-23} \mathrm{eV}}\right)^{1 / 2}\left\langle\left(\frac{M_{i j}^{*}}{M_{\mathrm{P}}}\right)^{2}\right\rangle,
$$

where the initial field value at the time oscillations begin, $M_{i j}^{*}$, is set by the misalignment mechanism. From a phenomenological point of view, the observed DM abundance $\Omega_{\mathrm{DM}}=0.26$ [8] can be matched for a wide range of $m_{\mathrm{FP}}$, independently of the presence of Hubble scale anisotropies [37] or primordial magnetic fields [39].

We remark on an important difference between oscillating scalar and spin-2 fields. Due to the intrinsic spin, the homogeneous modes of the latter are not isotropic but must respect the stringent microwave background anisotropies bound. However, similarly to the case of spin-1 field [40], the induced characteristic quadrupolar anisotropy is dynamically driven to negligible levels [7] by the rapid oscillations of the spin-2 field. Before oscillations begin, the anisotropy is inevitably present, but it is negligible since the energy density of $M_{\mu \nu}$ is vastly subdominant compared to that of other isotropic components.

BT can be extended to a multimetric gravity theory by introducing extra spin-2 fields and the corresponding interaction potentials, resulting in the most natural framework to implement the clockwork mechanism for gravity $[22,23]$. The basic idea behind this paradigm is to consider $N$ sites of identical physical systems, spin-2 sectors in our case, sequentially coupled to each other via interaction potentials which prevent the appearance of ghost modes. In the limit $N \rightarrow \infty$, the system then effectively acquires a continuous extra dimension. This construction aims to explain hierarchies in physics in terms of exponential dependences between physical parameters induced by the separation of the sites. In the multimetric clockwork 
interpretation of BT, the apparent weakness of gravity, or the unnaturally large value of the Planck scale, can then be explained as an exponential suppression of a TeV-scale fundamental mass parameter appearing in a distant site of the theory which directly interacts with matter.

As for the possible cosmological tests of our scenario, large scale structure formation casts a lower bound on the oscillating field mass $m_{\mathrm{FP}} \gtrsim 10^{-23} \mathrm{eV}$, that roughly corresponds to the inverse size of a dwarf galaxy [41,42]. Black hole superradiance, instead, excludes masses $6 \times 10^{-13} \mathrm{eV} \gtrsim m_{\mathrm{FP}} \gtrsim 2 \times 10^{-11} \mathrm{eV}$ independently of the origin of the radiated field [15], whereas pulsar timing arrays will probe in the future the lowest end of the available mass range [43]. Spin-2 masses $10^{-23} \mathrm{eV} \lesssim$ $m_{\mathrm{FP}} \lesssim 10^{-18} \mathrm{eV}$ can also be tested by seeking secular changes in the orbital period of binary pulsars [44], and with different systems lying on different orbital planes, it is in principle even possible to determine the spin-2 nature of the disturbance. The lowest end of the considered mass spectrum could also be constrained by measurements of the gravitational signal emitted during the inspiraling of binary systems, as well as by the requirement of perturbativity for the cosmological solutions of BT [29]: $\rho<m_{\mathrm{FP}}^{2} M_{\mathrm{P}}^{2} / \alpha^{2}$, where $\rho$ is the total energy density of the Universe. Supposing a radiation dominated regime, by recasting the bound in terms of the reheating temperature $T$, the requirement of successful big bang nucleosynthesis, $T>$ $\mathcal{O}(1) \mathrm{MeV}$, then implies $m_{\mathrm{FP}} \gtrsim 10^{-15} \sqrt{\alpha} \mathrm{eV}$. Clearly, depending on the value of $\alpha$, this consistency bound might supersede the limits discussed above. On the contrary, the implications of current observations within the parametrized post-Newtonian formalism are, in general, less stringent [45]. Notice that no delay between electromagnetic and gravitational signals [46-49] is produced in this scenario, since within our approximation there is only one, fully nonlinear, background metric, Eq. (9).

As is customary in ALPs scenarios, we attributed the initial displacement of our field to a misalignment mechanism, possibly related to a gravitational phase transition resulting in the interacting potential of Eq. (1). Alternatively, a nonzero displacement of the field can be induced by its random Gaussian fluctuations during the de Sitter inflationary epoch. In this case, we expect that [50] $M_{i j}^{*}<3 H_{\text {inf }} \sqrt{N}$, where $H_{\text {inf }} \sim 10^{14} \mathrm{GeV}$ is the Hubble value during inflation and $N$ is the total number of $e$-folds. This relation, together with Eq. (18), implies a lower bound $m_{\mathrm{FP}} \gtrsim 10^{-12} \mathrm{eV}$ which greatly restricts the available parameter space. In this case, the maximal spin-2 field coherence distances is about $10^{3} \mathrm{~km}$, yielding possible implications on small scale structure formation issues such as the core-vs-cusp problem [51]. The inflationary origin of the spin-2 field initial displacement, however, is strongly constrained by the current limits on isocurvature perturbations which the spin-2 field sources in the same way as
ALPs [19]: $\Delta_{\text {iso }} \approx H_{\text {inf }} / \pi M_{i j}^{*}$. Whereas for a generic misalignment mechanism the current measurements $\Delta_{\text {ad }} \sim$ $5 \times 10^{-5}$ [52] simply imply $M_{i j}^{*} \gtrsim 10^{-6} H_{\text {inf }} / \pi$, for an inflationary origin of the displacement, this bound translates into a harsh constraint on the total number of $e$-folds $N \gtrsim 10^{10}$.

As for clockwork multimetric gravity, we expect the theory to involve many spin- 2 massive fields, characterized by different Fierz-Pauli masses $m_{\mathrm{FP}}^{1}>m_{\mathrm{FP}}^{2}>\ldots>m_{\mathrm{FP}}^{N}$, which would start to oscillate at different times during the evolution of the Universe. As a result, depending on the initial displacement of the fields, the scenario predicts rapid changes in the energy density of the matter component of the Universe as the latter evolves. If the masses of (some of) the spin-2 fields are such that oscillations begin close to matter-radiation equality, $m_{\mathrm{FP}} \sim 10^{-23}$, future observations might detect the transitions eras when these fields start to contribute to the matter energy density [21].

Turning to laboratory experiments, the classical probes for new light fields are fifth force searches and tests of the equivalence principle, which constrain new theories independently of their link to DM.

Fifth force experiments seek deviations from the $1 / r$ scaling of the gravitational potential, induced in our case by the coupling $\alpha / M_{\mathrm{P}}$ of the massive spin-2 field to the stress-energy tensor of matter. By examining spherically symmetric solutions of BT, we predict a correction $\Delta \Phi_{N}=$ $\alpha^{2} \exp \left(-m_{\mathrm{FP}} r\right)$ to Newton's potential. The most sensitive experimental results [30,53] imply that $\alpha \leq 10$ for $m_{\mathrm{FP}} \sim$ $10^{-1} \mathrm{eV}$ and $\alpha<10^{-2}$ for $m_{\mathrm{FP}}<10^{-2} \mathrm{eV}$.

Tests of the equivalence principle are instead sensitive to variations of fundamental parameters of the Standard Model, such as the electron to proton mass ratio, $m_{e} / m_{p}$, and the elementary electric charge. Although in our case the massive spin-2 field couples to proton and electron masses identically, experiments [54] constrain $\alpha<10^{-1-2}$ for $10^{-23}<m_{\mathrm{FP}}<10^{-6} \mathrm{eV}$, covering most of the considered mass range [15].

Equivalence principle-violating effects could be also detectable as temporal and directional variations of the elementary electric charge [9], which induce modulations in the emission lines of atoms, molecules, and nuclei investigated by means of atomic and nuclear clocks, atomic spectroscopy, dedicated resonant mass detectors, laser interferometers, and ALP detectors. The massive spin-2 field we consider, in fact, interacts with radiation according to

$$
S \supset \frac{\alpha}{M_{\mathrm{P}}} M^{\mu \nu}\left(\frac{1}{4} \mathcal{G}_{\mu \nu} F^{\rho \sigma} F_{\rho \sigma}-F_{\mu}{ }^{\rho} F_{\nu \rho}\right),
$$

resulting in effective couplings to electric and magnetic field of the forms $E_{i} E_{j} \pm B_{i} B_{j}$ and $E_{i} B_{j} \pm B_{i} E_{j}$. Whereas the bounds from Refs. [10-19] apply here at least as an order of magnitude estimate, the explicit form of the spin- 2 coupling 
is clearly different from that of the scalar and pseudoscalar fields usually considered in the literature, which couple to electric and magnetic fields via $E^{2}-B^{2}$ and $E \cdot B$, respectively. The induced nontrivial polarization correlations and the possible directional and temporal variations of electric charge therefore constitute a distinguishing signature of the scenario.

Lastly, searches aimed at detecting photon-ALPs conversion in strong magnetic fields, as light-shining-throughthe-wall experiments for instance, probe this scenario with the same reach as in the usual ALPs model. In this case, the characteristic decay constant is to be compared to $\alpha / M_{\mathrm{P}}$.

We conclude that the oscillating massive spin-2 field is a viable new candidate for DM. While the current experiments and observations constrain a part of the parameter space of this scenario, there is plenty of room left for a future discovery. Most importantly, beside traditional tests of gravity, laboratory searches ranging from precision measurements of the fundamental electric charge with atomic clocks to dedicated resonant mass detectors, as well as experiments devoted to axionlike particles, could contribute in the proposed framework to unveil and comprehend the properties of gravity and dark matter.

\section{ACKNOWLEDGEMENT}

The authors thank Denis Comelli, Tomi Koivisto, Maxim Pospelov, Sabir Ramazanov, Yevgeny Stadnik, Mikael Von Strauss, and Ville Vaskonen for useful discussions. This research was financed by the Estonian Research Council Grants No. IUT23-6 and No. PUT 808 and by the European Union through the ERDF CoE Grant No. TK133. The calculation of the massive spin-2 field equations of motion over a Friedmann-Robertson-Walker background were performed with the support of the tensor manipulation Mathematica packages XTENSOR and XCOBA [55], developed by J.-M. Martín-García and D. Yllanes (http:// www.xact.es).
[1] G. Jungman, M. Kamionkowski, and K. Griest, Supersymmetric dark matter, Phys. Rep. 267, 195 (1996).

[2] G. Bertone, D. Hooper, and J. Silk, Particle dark matter: Evidence, candidates and constraints, Phys. Rep. 405, 279 (2005).

[3] T. Clifton, P. G. Ferreira, A. Padilla, and C. Skordis, Modified gravity and cosmology, Phys. Rep. 513, 1 (2012).

[4] S. F. Hassan and R. A. Rosen, Bimetric gravity from ghost-free massive gravity, J. High Energy Phys. 02 (2012) 126.

[5] A. Schmidt-May and M. von Strauss, Recent developments in bimetric theory, J. Phys. A 49, 183001 (2016).

[6] J. Preskill, M. B. Wise, and F. Wilczek, Cosmology of the invisible axion, Phys. Lett. 120B, 127 (1983).

[7] J. A. R. Cembranos, C. Hallabrin, A. L. Maroto, and S. J. N. Jareno, Isotropy theorem for cosmological vector fields, Phys. Rev. D 86, 021301 (2012).

[8] P. A. R. Ade et al. Planck 2015 results. XIII. Cosmological parameters, Astron. Astrophys. 594, A13 (2016).

[9] T. Damour and J.F. Donoghue, Equivalence principle violations and couplings of a light dilaton, Phys. Rev. D 82, 084033 (2010).

[10] A. Arvanitaki, J. Huang, and K. Van Tilburg, Searching for dilaton dark matter with atomic clocks, Phys. Rev. D 91, 015015 (2015).

[11] Y. V. Stadnik and V. V. Flambaum, Improved limits on interactions of low-mass spin-0 dark matter from atomic clock spectroscopy, Phys. Rev. A 94, 022111 (2016).

[12] K. Van Tilburg, N. Leefer, L. Bougas, and D. Budker, Search for Ultralight Scalar Dark Matter with Atomic Spectroscopy, Phys. Rev. Lett. 115, 011802 (2015).

[13] A. Hees, J. Guéna, M. Abgrall, S. Bize, and P. Wolf, Searching for an Oscillating Massive Scalar Field as a Dark
Matter Candidate using Atomic Hyperfine Frequency Comparisons, Phys. Rev. Lett. 117, 061301 (2016).

[14] Y. V. Stadnik and V. V. Flambaum, Searching for Dark Matter and Variation of Fundamental Constants with Laser and Maser Interferometry, Phys. Rev. Lett. 114, 161301 (2015).

[15] A. Arvanitaki, S. Dimopoulos, and K. Van Tilburg, Sound of Dark Matter: Searching for Light Scalars with ResonantMass Detectors, Phys. Rev. Lett. 116, 031102 (2016).

[16] Y. V. Stadnik and V. V. Flambaum, Enhanced effects of variation of the fundamental constants in laser interferometers and application to dark matter detection, Phys. Rev. A 93, 063630 (2016).

[17] G. G. Raffelt, Astrophysical methods to constrain axions and other novel particle phenomena, Phys. Rep. 198, 1 (1990).

[18] A. Ringwald, Exploring the role of axions and other WISPs in the dark universe, Phys. Dark Universe 1, 116 (2012).

[19] D. J. E. Marsh, Axion cosmology, Phys. Rep. 643, 1 (2016).

[20] K. Hinterbichler and R. A. Rosen, Interacting spin-2 fields, J. High Energy Phys. 07 (2012) 047.

[21] L. Amendola et al., Cosmology and fundamental physics with the euclid satellite, arXiv:1606.00180.

[22] D. E. Kaplan and R. Rattazzi, Large field excursions and approximate discrete symmetries from a clockwork axion, Phys. Rev. D 93, 085007 (2016).

[23] G. F. Giudice and M. McCullough, A clockwork theory, J. High Energy Phys. 02 (2017) 036.

[24] D. G. Boulware and S. Deser, Can gravitation have a finite range?, Phys. Rev. D 6, 3368 (1972).

[25] C. de Rham, G. Gabadadze, and A. J. Tolley, Resummation of Massive Gravity, Phys. Rev. Lett. 106, 231101 (2011).

[26] Y. Yamashita, A. De Felice, and T. Tanaka, Appearance of Boulware-Deser ghost in bigravity with doubly coupled matter, Int. J. Mod. Phys. D 23, 1443003 (2014). 
[27] C. de Rham, L. Heisenberg, and R. H. Ribeiro, On couplings to matter in massive (bi-)gravity, Classical Quantum Gravity 32, 035022 (2015).

[28] E. Babichev, L. Marzola, M. Raidal, A. Schmidt-May, F. Urban, H. Veermäe, and M. von Strauss, Bigravitational origin of dark matter, Phys. Rev. D 94, 084055 (2016).

[29] E. Babichev, L. Marzola, M. Raidal, A. Schmidt-May, F. Urban, H. Veermäe, and M. von Strauss, Heavy spin-2 dark matter, J. Cosmol. Astropart. Phys. 09(2016) 016.

[30] J. Murata and S. Tanaka, A review of short-range gravity experiments in the LHC era, Classical Quantum Gravity 32, 033001 (2015).

[31] C. M. Will, The confrontation between general relativity and experiment, Living Rev. Relativity 17, 4 (2014).

[32] M. S. Volkov, Cosmological solutions with massive gravitons in the bigravity theory, J. High Energy Phys. 01 (2012) 035 .

[33] M. von Strauss, A. Schmidt-May, J. Enander, E. Mortsell, and S. F. Hassan, Cosmological solutions in bimetric gravity and their observational tests, J. Cosmol. Astropart. Phys. 03 (2012) 042.

[34] D. Comelli, M. Crisostomi, F. Nesti, and L. Pilo, FRW cosmology in ghost free massive gravity, J. High Energy Phys. 03 (2012) 067; Erratum, J. High Energy Phys. 06 (2012) 020(E).

[35] M. Berg, I. Buchberger, J. Enander, E. Mortsell, and S. Sjors, Growth histories in bimetric massive gravity, J. Cosmol. Astropart. Phys. 12 (2012) 021.

[36] Y. Akrami, T. S. Koivisto, and M. Sandstad, Accelerated expansion from ghost-free bigravity: A statistical analysis with improved generality, J. High Energy Phys. 03 (2013) 099.

[37] K.-i. Maeda and M. S. Volkov, Anisotropic universes in the ghost-free bigravity, Phys. Rev. D 87, 104009 (2013).

[38] K. Aoki and S. Mukohyama, Massive gravitons as dark matter and gravitational waves, Phys. Rev. D 94, 024001 (2016).

[39] K. Aoki and K.-i. Maeda, Condensate of massive graviton and dark matter, arXiv:1707.05003.

[40] A. E. Nelson and J. Scholtz, Dark light, dark matter and the misalignment mechanism, Phys. Rev. D 84, 103501 (2011).

[41] R. Hlozek, D. Grin, D. J. E. Marsh, and P. G. Ferreira, A search for ultralight axions using precision cosmological data, Phys. Rev. D 91, 103512 (2015).
[42] L. Hui, J. P. Ostriker, S. Tremaine, and E. Witten, Ultralight scalars as cosmological dark matter, Phys. Rev. D 95, 043541 (2017).

[43] A. Khmelnitsky and V. Rubakov, Pulsar timing signal from ultralight scalar dark matter, J. Cosmol. Astropart. Phys. 02 (2014) 019.

[44] D. Blas, D. L. Nacir, and S. Sibiryakov, Ultralight Dark Matter Resonates with Binary Pulsars, Phys. Rev. Lett. 118, 261102 (2017).

[45] M. Hohmann, Post-Newtonian parameter and the deflection of light in ghost-free massive bimetric gravity, Phys. Rev. D 95, 124049 (2017).

[46] B. P. Abbott et al., Gravitational waves and gamma-rays from a binary neutron star merger: GW170817 and GRB 170817A, Astrophys. J. 848, L13 (2017).

[47] H. Wang et al., GW170817/GRB 170817A/AT2017gfo association: some implications for physics and astrophysics, arXiv:1710.05805.

[48] J.-J. Wei, X.-L. Fan, B.-B. Zhang, X.-F. Wu, H. Gao, P. Mészáros, B. Zhang, Z.-G. Dai, S.-N. Zhang, and Z.-H. $\mathrm{Zhu}$, Multimessenger tests of the weak equivalence principle from GW170817 and its electromagnetic counterparts, J. Cosmol. Astropart. Phys. 11 (2017) 035.

[49] S. Boran, S. Desai, E. Kahya, and R. Woodard, GW170817 falsifies dark matter emulators, arXiv:1710.06168.

[50] J. R. Espinosa, G. F. Giudice, E. Morgante, A. Riotto, L. Senatore, A. Strumia, and N. Tetradis, The cosmological Higgstory of the vacuum instability, J. High Energy Phys. 09 (2015) 174.

[51] W. J. G. de Blok, The core-cusp problem, Adv. Astron. 2010, 789293 (2010).

[52] P. A. R. Ade et al., Planck 2015 results. XX. Constraints on inflation, Astron. Astrophys. 594, A20 (2016).

[53] D. J. Kapner, T. S. Cook, E. G. Adelberger, J. H. Gundlach, B. R. Heckel, C. D. Hoyle, and H. E. Swanson, Tests of the Gravitational Inverse-Square Law Below the Dark-Energy Length Scale, Phys. Rev. Lett. 98, 021101 (2007).

[54] S. Schlamminger, K. Y. Choi, T. A. Wagner, J. H. Gundlach, and E. G. Adelberger, Test of the Equivalence Principle using a Rotating Torsion Balance, Phys. Rev. Lett. 100, 041101 (2008).

[55] D. Brizuela, J. M. Martin-Garcia, and G. A. M. Marugan, xPert: Computer algebra for metric perturbation theory, Gen. Relativ. Gravit. 41, 2415 (2009). 